\title{
Practice-Based Comparison of Imaging Methods for Visualization of Toolmarks on an Egyptian Scarab
}

\author{
Lindsay MacDonald ${ }^{1}$, Maria Filomena Guerra ${ }^{2}$, Ruven Pillay ${ }^{3}$, Mona Hess ${ }^{1}$, \\ Stephen Quirke ${ }^{4}$, Stuart Robson ${ }^{1}$, and Ali Hosseininaveh Ahmadabadian ${ }^{1}$ \\ ${ }^{1}$ Dept. of Civil, Environmental and Geomatic Engineering, \\ University College London, London, UK \\ (1.macdonald, m.hess, s.robson, ali.ahmadabadian.10) @ucl .ac.uk \\ ${ }^{2}$ UMR 8096 CNRS, France \\ maria-filomena.guerra@mae.u-paris10.fr \\ ${ }^{3}$ C2RMF, Musée du Louvre, Paris, France \\ ruven.pillay@culture.gouv.fr \\ ${ }^{4}$ Institute of Archaeology, University College London, London, UK \\ s.quirke@ucl.ac.uk
}

\begin{abstract}
D representations were made of a small Egyptian scarab with a gold band by a number of methods, based on photogrammetry and photometric stereo. They were evaluated for colour fidelity and spatial detail, in the context of a study of toolmarks and manufacturing techniques of jewellery in ancient Egypt. It was found that although a 3D laser scanner gave the best geometric accuracy, the camera-based methods of photogrammetry and photometric stereo gave better representation of fine detail and colour on the object surface.
\end{abstract}

Keywords: Digital heritage, image acquisition, 3D imaging, visualisation, gold.

\section{Introduction and Research Scope}

Little is known about Egyptian jewellery dated to the Bronze Age and the practices of the goldsmith. With the exception of studies by Schorsch [1] and Lucas [2], the main analytical researches carried out until recently concerned the collections of the Metropolitan Museum of New York: jewellery from the burial of Wah [3], jewellery from the burial of the foreign wives of Thutmose III [4], and a large set of aurian silver objects from the Ashmolean Museum of Oxford [5]. As a result, neither the goldsmithing practices in Ancient Egypt - alloys and manufacturing technologies - nor the origin of the gold was understood. In 2009 however, two multi-disciplinary studies of the Ancient Egypt collections of the National Museums Scotland [6,7] shed more light on this jewellery and raised new questions on a very particular period of Egyptian history, the 2nd Intermediate Period (1650-1550 BC). This led to multidisciplinary studies of other collections, including the British Museum [8], which applied elemental analysis to determine the composition of alloys, the origin of the gold and the evolution of practices for joining and decorating the objects.

The CNRS PICS project 5995 entitled Study of Bronze Age Egyptian Gold Jewellery has involved exchanges between several French and UK institutions. The focus is on the origin of gold and composition of the alloys used for forming, soldering, and 
decorating, in order to provide new evidence on the technologies used in the Egyptian workshops producing gold work during the Bronze Age. Several objects from the collections of the museum partners were studied by science-based techniques, including optical microscopy, X-radiography, scanning electron microscopy, X-ray fluorescence (XRF), and ion beam analysis (particularly PIXE and PIGE).

The analysis carried out on items attributed to the 2nd Intermediate Period and the New Kingdom allowed some unexpected practices to be demonstrated, such as the coexistence of very thin together with very thick hard-soldering; the use of casting even for very small items; the reuse without re-melting of some smaller elements; and the almost continuous use of placer gold. Evidence was also found for polychrome effects by using not only different materials but also several gold alloys with different copper and silver content. The latter necessitates an effective method for reproducing the colours present in the object - knowing that some composite items show strong surface alterations caused by atmospheric corrosion, which 'hide' their original colours.

The construction of an object is influenced by the decorative techniques, evidenced by the presence of tool marks and wear-marks giving indications about the object's function and possible re-use. Analysis requires relevant imaging of high quality, at different scales and depths, such as optical microscopy, scanning electron microscopy and $\mathrm{X}$ radiography $[9,10]$. For this reason, we wished to investigate the contribution of $3 \mathrm{D}$ imaging acquisition with $3 \mathrm{D}$ reconstruction to represent the original colours and surface detail of the materials used in the production of Egyptian gold jewellery.

\section{Scarab: XRF Analysis}

We selected an object that is very representative of Egyptian production, a small scarab (Fig. 1) of engraved steatite with a gold band, from the UCL Petrie Museum of Egyptian Archaeology (reference UC11365). Dated to the Late Middle Kingdom (1850-1750 BC) it is inscribed in hieroglyphs on the underside with the personal name and title "estate overseer of the granary Iufseneb" within the scroll border.
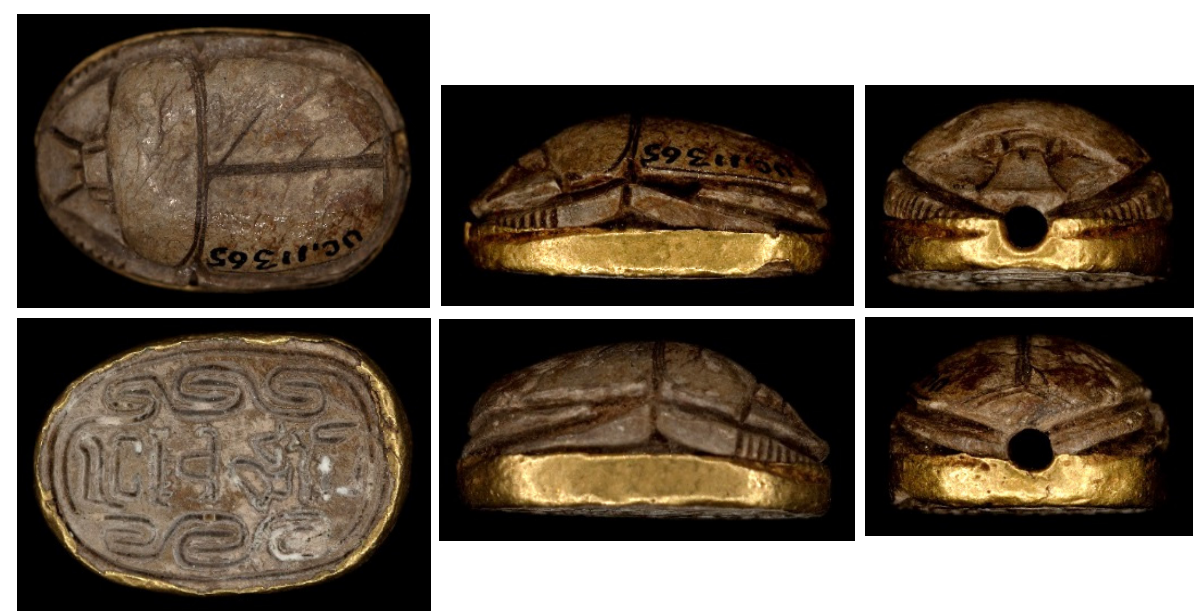

Fig. 1. Six views of Scarab (UC11365). Dimensions 26.7 (W) x 18.6 (H) x 11.4 (D) mm 
The aim of this study was to investigate the capabilities of different methods of 3D imaging to represent the original colours and surface details for visual inspection of toolmarks and other material properties. The scarab poses particular challenges for imaging due to its characteristics: a small object, with finely engraved inscription, the back is curved and polished to a semi-gloss finish, and the encircling chased gold band has a high specularity. Specific objectives were:

- To produce a 3D representation with a resolution high enough to visualize tool marks, typically about 100 microns in dimension;

- To display detailed surface geometry and correct colour;

- To evaluate whether transport of imaging equipment to museums abroad is feasible, and repeatable enough to produce successful imaging results;

- To compare imaging outcomes with established but costly and timeintensive analytic methodologies in dedicated laboratories.

The scarab's inscribed gold band was analysed by handheld XRF together with three gold alloy standards, whose expected and measured contents are presented in Table 1. Their good agreement is clear. Analysis of four different regions on the scarab showed that on average the alloy is composed of $0.3 \% \mathrm{Cu}, 5.7 \% \mathrm{Ag}$ and 94.3 $\% \mathrm{Au}$, which is consistent with expectations for the period of attribution.

Table 1. Compositional data obtained by handheld XRF for inscribed gold band on scarab

\begin{tabular}{|ccccc|}
\hline & & Cu wt \% & Ag wt \% & Au wt \% \\
\hline Standard 1 & reference & 1.1 & 6.4 & 92.5 \\
& measured & 1.1 & 6.3 & 92.6 \\
\hline Standard 2 & reference & 4.1 & 4.6 & 91.3 \\
& measured & 4.4 & 4.3 & 91.2 \\
\hline Standard 3 & reference & 4.0 & 4.0 & 92.0 \\
& measured & 4.2 & 4.2 & 91.6 \\
\hline Scarab UC11365 & average & 0.3 & 5.7 & 94.3 \\
& $\sigma$ & 0.1 & 0.02 & 0.1 \\
\hline
\end{tabular}

\section{3D Acquisition Methods Used for the Scarab}

\subsection{Photogrammetry \#1: C2RMF Software Method}

Photographic images were acquired by placing the scarab on a rigid black tray, which incorporated a number of retro-reflective targets for geometric calibration. Around 80 images were taken using a Nikon D3200 camera with a Nikkor $105 \mathrm{~mm}$ macro lens, from various angles by rotating the tray, and by turning the scarab onto its side. Flash lighting was diffused by soft boxes and reflectors pointed towards the ceiling. A standard photogrammetry pipeline was employed, consisting of an initial calibration of the images for both photometric and geometric distortion. Photometric/colorimetric calibration was performed through the use of a standard colour chart and correcting for noise, linearity and colour response. Images were undistorted by estimating parameters from the camera's chip size and focal length. 

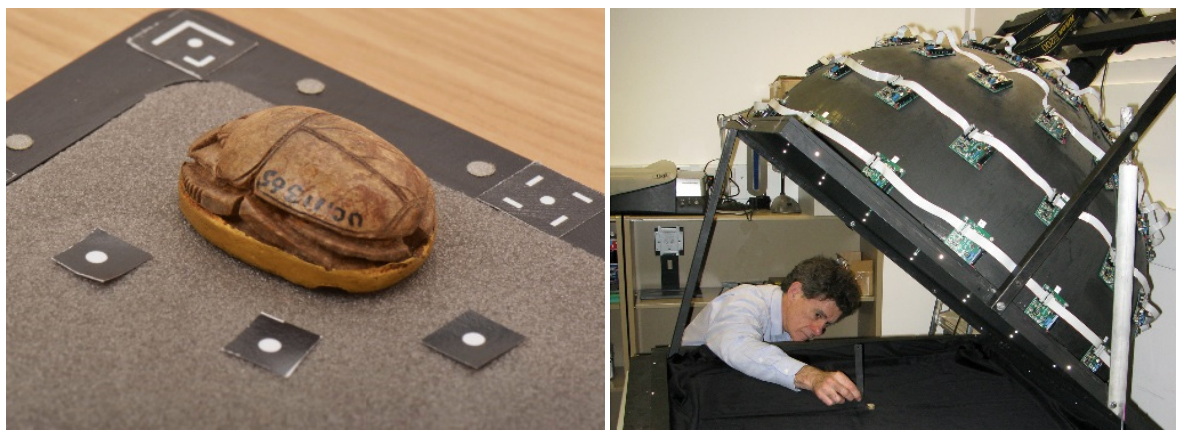

Fig. 2. (left) Photogrammetric imaging setup with scarab on target board; (right) positioning the scarab on the baseboard of the UCL dome

The open source Bundler software [11] was used to derive structure from the images with optimisation through bundle adjustment [12].This result was used to produce a dense 3D reconstruction using open source PMVS-CMVS multi-view stereo software [13]. The resulting output is a dense point cloud of the scene. As the scarab needed to be repositioned during acquisition in order to obtain images of the whole object, each 'scene' was reconstructed separately, then cleaned and fused together during post-processing. Although the resulting 3D geometry is less accurate than that of a laser scanner, photogrammetry-based techniques enable the acquisition of accurate colour data, which is essential for cultural heritage applications. If a mesh surface is reconstructed from the point cloud, the texture obtained from the imagery can then be reprojected onto the surface giving a high resolution texture even on a lower resolution geometry.

\subsection{Photogrammetry \#2: UCL Software Method}

The same image set was processed with an in-house software pipeline at UCL. A dense and accurate point cloud was generated [14]. After geometrically correcting ('undistorting') the images, the corresponding measurements extracted from the images were used to compute approximate 3D coordinates in Bundler. A photogrammetric bundle adjustment with the relative orientation parameters of each camera position was compared with the calibrated baseline to estimate a scale factor, which was applied on the camera locations and 3D coordinates. After resolving the scale, these data were input into the PMVS processing software VisualSFM to generate a dense point cloud.

\subsection{D Colour Laser Scanning}

The Arius 3D colour laser scanner at UCL is used for digitisation of museum objects, and for traceable and repeatable production of metric surface models. It delivers 3D RGB point data at a sampling interval of $0.1 \mathrm{~mm}$ (100 microns) at an accuracy of c. $0.025 \mathrm{~mm}$ (25 microns) over the object surface [15]. Twenty-six separate scans were made, with the scarab turned to many different positions to ensure that all surfaces were captured. The corresponding point clouds were processed in the Pointstream software to produce a unified 3D representation with approximately 402,000 points. 


\subsection{Reflectance Transform Imaging}

Six sets of 64 images were taken in the UCL dome imaging system, with the scarab in six different orientations (Fig. 1), using a Nikon D200 camera with Nikkor $200 \mathrm{~mm}$ macro lens. All images in each set were taken from the fixed camera viewpoint, i.e. vertically from above, so all were in pixel register, but each was illuminated from a different direction over the hemisphere. Each image set was used to construct a representation of the scarab by variants of the polynomial texture map (PTM) technique [16], fitting a biquadratic function (6 coefficients) and hemispherical harmonics (16 coefficients) to the intensity distribution at each point. In addition a new model was developed, fitting a Lorentzian function to the specular component (Fig. 3).

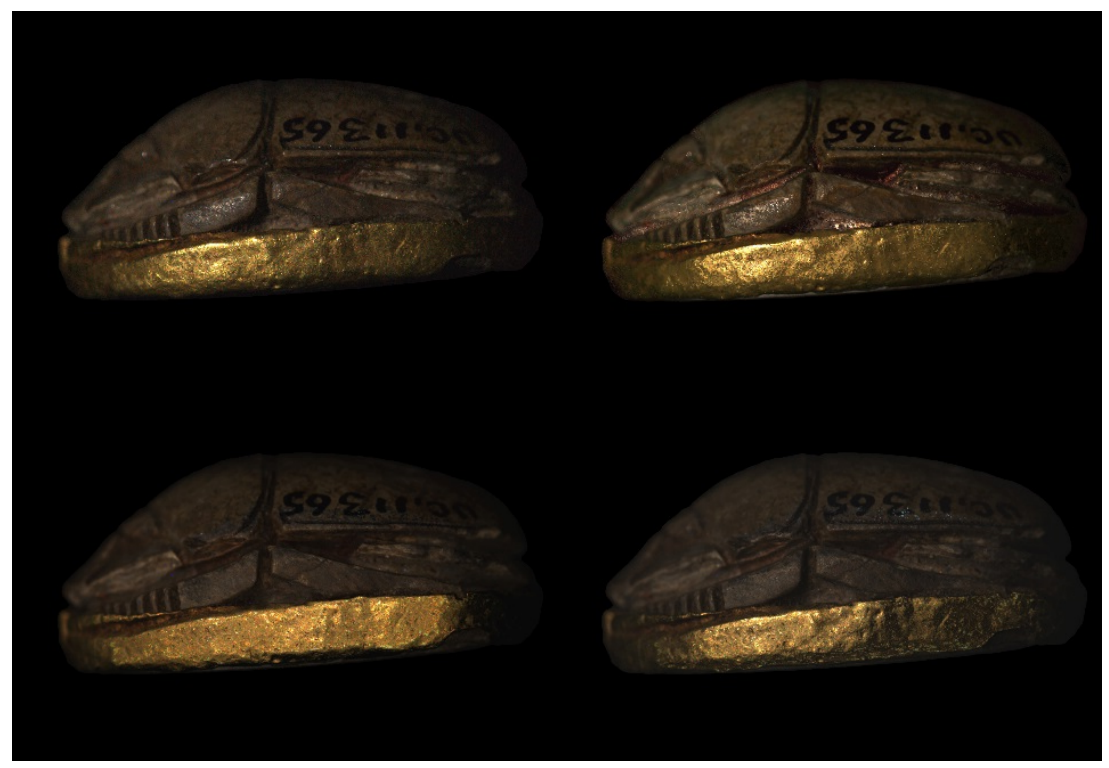

Fig. 3. Screenshots from the custom software viewer showing specularity of gold in four representations of the left side of the scarab: (top left) original image; (bottom left) PTM model; (bottom right) hemispherical harmonics (HSH) model; (top right) Lorentzian specular model

\subsection{Photometric Stereo}

The same six sets of images from the UCL dome were used to reconstruct the 3D surface by photometric stereo [17], by determining the surface normal at each pixel and then integrating them into a consistent surface. A new adaptive method was employed developed to find an optimal subset of intensities from all 64 candidates at each pixel, by sorting them into order and selecting a range between the shadow and specular regions, followed by regression over this subset for an accurate estimate of the normal. The height field was reconstructed as a digital terrain map by projecting the surface gradients onto Fourier basis functions to ensure integrability [18], then exported as a point cloud for 3D visualisation. 


\section{$4 \quad$ Evaluation of Imaging Methods}

The various visual representations were evaluated at UCL by the domain expert (MFG), who was interested in the visibility of toolmarks and other material treatments. The models produced by the three RTI representations were displayed in a custom software viewer, and point clouds generated by the other four methods in the Pointstream viewer. The visual inspection was directly related to analytical procedures in the CNRS lab, such as stereomicroscopic images at 30-50X magnification or with a Scanning Electron Micro scope (SEM). The advantages of the stereomicroscope would be images with colour rendition, but restricted to only one viewpoint. The advantage of the SEM would be a very detailed rendering of the surface with toolmarks, but without colour.
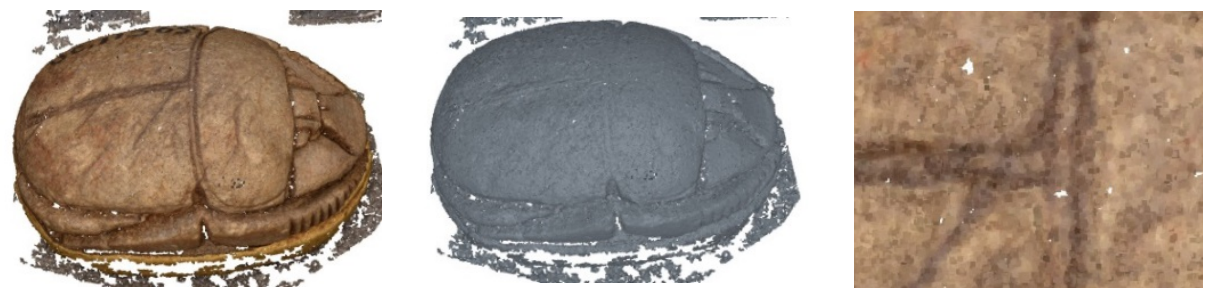

Fig. 4. (left) Photogrammetry \#2 with and without colour. While the colour is close to original object due to colour control, the method produced a significant quantity of holes and surface noise; (right) Detail of scarab top, showing erroneous cut by craftsman.

Both photogrammetric methods showed realistic colour and significant detail I (Fig. 4), with a resolution of approximately 20 pixels $/ \mathrm{mm}$ (50 micron sampling). The geometry was overall accurate but was compromised by holes from missing data due to specular reflection from the gold during the acquisition process. There was also a noticeable level of surface noise in the reconstruction.
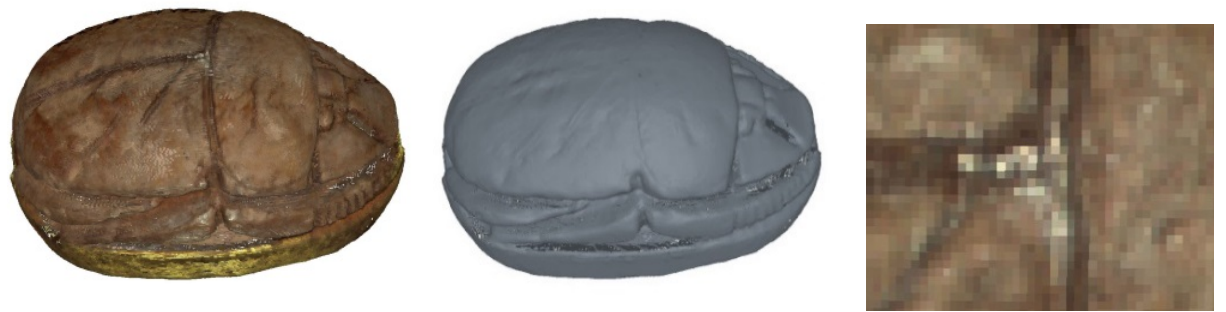

Fig. 5. (left) 3D reconstruction from the point cloud generated by the Arius laser scanner, showing the redness caused by the non-optimal laser wavelengths; (right) Detail of back

The researcher showed interest in the geometric accuracy of the 3D colour laser scan, but was not impressed by the rendition of surface detail (Fig. 5). Even though the scanner was expected to show sufficient details with a sampling pitch of 100 microns (10 points $/ \mathrm{mm}$ ), it did not meet the expectation, and revealed less detail than shown by magnification with a hand lens. The finest surface feature that can be wrought on an artefact by a craftsman is approximately 50 micron, to capture which the scanner should 
have a resolution of at least 25 micron (i.e. 40 pixels $/ \mathrm{mm}$ ) [19]. Moreover the rendering of surface colour by the three lasers is not so accurate as with a camera, because the monochromatic sampling of the reflectance spectrum leads to severe metamerism [20].

The specular rendering in its custom written viewer was successful (Fig. 3). The lighting and display is standardised and as such is repeatable and traceable. The opportunity to display diffuse colour and specular reflectance separately made it easier to identify tool marks and material striations. The original capture resolution in the images was 65 pixels $/ \mathrm{mm}$ ( 15 micron) but this was reduced to 53 pixels $/ \mathrm{mm}$ (19 micron) to fit the full outline of the scarab into the HD display window. The rendition of the photometric stereo in 3D did not provide a completely metric image in the round (Fig. 6). But was successful, especially when reading the hieroglyphs on the underside of the scarab. The amount of detail that could be deciphered through both visualisations was key to conclude that it might replace long processes that might only bring out details.
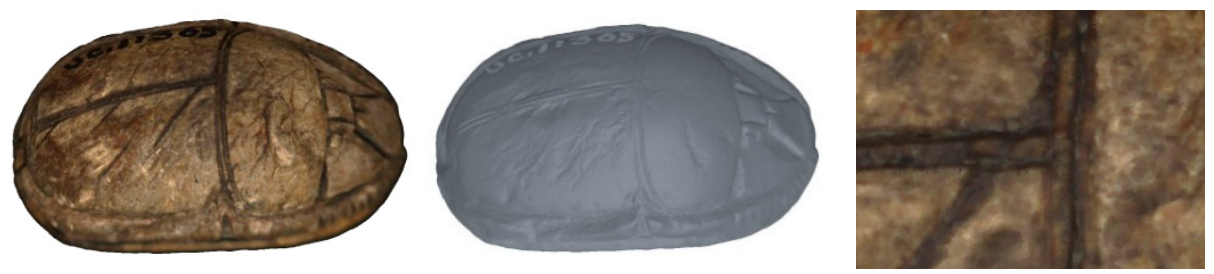

Fig. 6. (left) The 3D reconstruction from photometric stereo produced a very dense point cloud with outstanding detail of surface features, which enabled the researcher to interpret the object

The results of the qualitative evaluation are summarised in Table 2. The laser scanner gave results that were excellent in terms of geometric accuracy, because of the precision of its coordinate measuring machine (CMM). But its results were inferior in both colour and resolution to the other techniques which derived 3D from sets of photographic images. For all methods the time required to set up and digitise the object was typically about an hour, but in all cases it took a day or more of operator time and/or computing time to process the data to produce the final 3D representation. For representing gloss and specularity, only the RTI method that modelled the diffuse and specular components separately produced acceptable results. In terms of portability the photogrammetric methods were best by far, because they required only a camera and board with targets affixed, whereas the RTI methods relied on the dome structure to provide the camera support and multiple sources of illumination.

Table 2. Qualitative comparison of 3D representations

\begin{tabular}{|c|c|c|c|c|c|c|}
\hline Technology & Colour & Specularity & Geometry & Resolution & Cost & Portability \\
\hline $\begin{array}{c}\text { Photogrammetry, } \\
\text { methods \#1 and \#2 }\end{array}$ & Good & Poor & Good & High & Low & Good \\
\hline $\begin{array}{c}\text { 3D colour laser } \\
\text { scanning }\end{array}$ & Poor & Poor & Excellent & Medium & High & Impossible \\
\hline $\begin{array}{c}\text { Reflectance trans- } \\
\text { form imaging }\end{array}$ & Good & Moderate & Poor & High & Medium & Poor \\
\hline Photometric stereo & Good & Excellent & Poor & High & Medium & Poor \\
\hline
\end{tabular}


Acknowledgments. This study was performed under the scope of CNRS PICS project 5995 'Analytical Study of Bronze Age Egyptian Gold Jewellery'. It was facilitated by the European COST Action TD1201 Colour and Space in Cultural Heritage $(\mathrm{COSCH})$.

\section{References}

1. Schorsch, D.: Precious-Metal Polychromy in Egypt in the Time of Tutankhamun. Journal of Egyptian Archaeology 87, 55-71 (2001)

2. Lucas, A., Harris, J.R.: Ancient Egyptian Materials and Industries. Edward Arnold, London (1962)

3. Schorsch, D.: The gold and silver necklaces of Wah. In: Brown, et al. (eds.) Conservation in Ancient Egyptian Collections, pp. 127-135. Archetype Publications, London (1995)

4. Lilyquist, C.: The tomb of the three foreign wives of Tuthmosis III. The Metropolitan Museum of Art, New York (2003)

5. Gale, N.H., Stos-Gale, Z.A.: Ancient Egyptian Silver. JEA 67, 103-115 (1981)

6. Tate, J., Eremin, K., Troalen, L.G., Guerra, M.F., Goring, E., Manley, B.: The 17th Dynasty gold necklace from Qurneh, Egypt. ArchéoScience 33, 121-128 (2009)

7. Troalen, L.G., Guerra, M.F., Tate, J., Manley, B.: Technological study of gold jewellery pieces dating from Middle to New Kingdom in Egypt. ArchéoScience 33, 111-119 (2009)

8. Miniaci, G., La Niece, S., Guerra, M.F., Hacke, M.: Analytical study of first royal Egyptian heart-scarab, Sobekemsaf. British Museum Technical Research Bulletin 7, 53-60 (2013)

9. Guerra, M.F.: An overview on the ancient goldsmith's skill and the circulation of gold in the past: The role of X-ray based techniques. X-Ray Spectrom 37, 317-327 (2008)

10. Guerra, M.F.: Role of radiation physics in the study and authentication of ancient gold work. Radiation Physics and Chemistry 95, 356-361 (2014)

11. Snavely, N., Seitz, S.M., Szeliski, R.: Photo Tourism: Exploring image collections in 3D. ACM Transactions on Graphics 25(3), 835-846 (2006)

12. Lourakis, M.I.A., Argyros, A.A.: The Design and Implementation of a Generic Sparse Bundle Adjustment Software Package. Tech. Rep. 340, FORTH, Heraklion, Crete (2004)

13. Furukawa, Y., Ponce, J.: Accurate, Dense, and Robust Multi-View Stereopsis. In: IEEE Conf. on Computer Vision and Pattern Recognition, vol. 32(8), pp. 1362-1376 (2007)

14. Ahmadabadian, A.H., Robson, S., Boehm, J., Shortis, M.: Image Selection in photogrammetric multi-view stereo methods for 3D reconstruction. In: Proc. SPIE, vol. 8791 (2013)

15. Hess, M., Robson, S.: 3D colour imaging for cultural heritage artefacts. In: Proc. ISPRS, XXXVIII (Part 5), pp. 288-292 (2010)

16. Malzbender, T., Gelb, D., Wolters, H.: Polynomial Texture Maps. Proc. ACM Siggraph 28, 519-528 (2001)

17. MacDonald, L.W., Robson, S.: Polynomial Texture Mapping and 3D Representations. In: Proc. ISPRS Commission V Symp. 'Close Range Image Measurement Techniques' (2010)

18. Frankot, R.T., Chellappa, R.A.: Method for Enforcing Integrability in Shape from Shading Algorithms. IEEE Trans. PAMI 10(4), 439-451 (1988)

19. MacDonald, L.W.: The Limits of Resolution. In: Proc. BCS Conf. on Electronic Visualisation and the Arts (EVA), London, pp. 149-156 (2010)

20. MacDonald, L.W.: Choosing Optimal Wavelengths for Colour Laser Scanners. In: Proc. 19th IS\&T/SPIE Color Imaging Conf., San Jose, pp. 357-362 (2011) 